\title{
Dynamic Response Analyses for Human-Induced Lateral Vibration on Congested Pedestrian Bridges
}

\author{
M. Yoneda
}

\begin{abstract}
In this paper, a lateral walking design force per person is proposed and compared with Imperial College test results. Numerical simulations considering the proposed walking design force which is incorporated into the neural-oscillator model are carried out placing much emphasis on the synchronization (the lock-in phenomenon) for a pedestrian bridge model with the span length of 50 $\mathrm{m}$. Numerical analyses are also conducted for an existing pedestrian suspension bridge. As compared with full scale measurements for this suspension bridge, it is confirmed that the analytical method based on the neural-oscillator model might be one of the useful ways to explain the synchronization (the lock-in phenomenon) of pedestrians being on the bridge.
\end{abstract}

Keywords - Pedestrian bridge, human-induced lateral vibration, neural-oscillator, full scale measurement, dynamic response analysis.

\section{INTRODUCTION}

$\mathrm{T}_{\mathrm{b}}$ HE phenomenon of synchronous lateral vibration induced by human walking on pedestrian bridges such as the London Millennium Bridge has increasingly attracted public attention [1]. Up to date, this kind of dynamic vibration has not led to structural failure of the bridge, but has often caused discomfort for the users and the temporary closure of the pedestrian bridges in order to provide proper countermeasures such as tuned mass damper. This lateral excitation phenomenon, for example the triggering of the lock-in phenomenon, has not been fully examined or modeled [2]. On the other hand, locomotion of animals, such as walking and running, is generated and controlled by the central nervous system which is called as the central pattern generator (CPG). Recently, the CPG framework has been widely utilized to develop locomotion controllers for autonomous walking robots. Therefore, a neural-oscillator model is adopted to investigate the human-induced lateral vibration on congested pedestrian bridges.

A design formula of $F=300 v$ is well known for the lateral walking forces. But, it must be noted that the lateral walking force due to this formula cannot be generated if the initial deck velocity is not given. Therefore, the new evaluation formula which is proportional to the square value of the deck velocity is also proposed in order to convert the neural-oscillator output to the pedestrian lateral force. Moreover, dynamic response analyses taking into account the neural oscillator is carried out for the pedestrian bridge model with the span length of $50 \mathrm{~m}$, placing much emphasis on the lock-in phenomenon from the

M. Yoneda (Professor) is with the Department of Civil and Environmental Engineering, Kindai University, Higashiosaka, Osaka 577-8502 Japan (phone: +81-6-6721-2332, fax: +81-6-6730-1320, e-mail: yoneda@ civileng.kindai.ac.jp). bridge design viewpoint. Numerical analyses are also carried out for an existing pedestrian suspension bridge with the center span of $62.4 \mathrm{~m}$.

\section{NEURAL-OSCILLATOR MODEL AND BEHAVIOR}

\section{A. Neural-Oscillator Model}

As shown in Fig. 1, the neural-oscillator model consists of two simulated neurons arranged in mutual inhibition. The model for the neuron proposed by Matsuoka [3] is adopted in this study. The firing rate of a real biological neuron with self-inhibition is governed by the following equations.

$$
\begin{gathered}
\tau_{1} \dot{x}_{1}=-x_{1}-\beta v_{1}-\gamma\left[x_{2}\right]^{+}+c-\left[u_{f}\right]^{+} \\
\tau_{2} \dot{v}_{1}=-v_{1}+\left[x_{1}\right]^{+} \\
\tau_{1} \dot{x}_{2}=-x_{2}-\beta v_{2}-\gamma\left[x_{1}\right]^{+}+c+\left[u_{f}\right] \\
\tau_{2} \dot{v}_{2}=-v_{2}+\left[x_{2}\right]^{+} \\
{\left[x_{2}\right]^{+}=\max (x, 0)} \\
y_{\text {out }}=y_{1}-y_{2}=\max \left(x_{1}, 0\right)-\max \left(x_{2}, 0\right)
\end{gathered}
$$

where $x_{i}$ is the firing rate, $v_{i}$ is a variable representing the self-inhibition of the neuron (modulated by the adaptation constant $\beta$ ), and the mutual inhibition is under the control of the parameter $\gamma$. The output of each neuron $y_{i}$ is employed as the positive part of $x_{i}$, and the output of the whole oscillator is expressed as $y_{\text {out }} . u_{f}$ is the input applied to the oscillator. The tonic excitation $c$ determines the amplitude of the oscillation in proportion to the amplitude of $c$. The oscillation is not occurred in case of $c=0$. The two time constants $\tau_{1}$ and $\tau_{2}$ determine the speed and shape of the oscillator output. $\tau_{1} / \tau_{2}$ should be in the range of $0.1 \sim 0.5$ for stable oscillation, [4].

\section{B. Neural-Oscillator Behavior}

When input is not applied to the neural-oscillator, it oscillates at a natural frequency, as shown in Fig. 2. In the case of $\beta=2.5, \gamma=2.5$ and $c=1.5$, natural frequency of the oscillator is calculated under changing two time constants $\tau_{1}$ and $\tau_{2}$. Fig. 3 shows the output frequency of the oscillator 
plotted against $1 / \tau_{1}$. Based on this figure, it can be revealed that the oscillator frequency is proportion to the magnitude of $1 / \tau_{1}$ under the condition that the ratio of $\tau_{1} / \tau_{2}$ is constant [5]. Therefore, the natural frequency of the neural- oscillator model in the case of $\beta=2.5, \gamma=2.5$ and $c=1.5$ (CASE-252515) can be evaluated by adopting the proposed equation, as shown in Table I. The proposed equation to evaluate the natural frequency where $\beta=1.5, \gamma=1.5, c=1.5$ (CASE-151515), is also shown in Table II. Comparing the results of Tables I and II, it is supposed that the natural frequency of the oscillator slightly depend on the values of $\beta$ and $\gamma$.

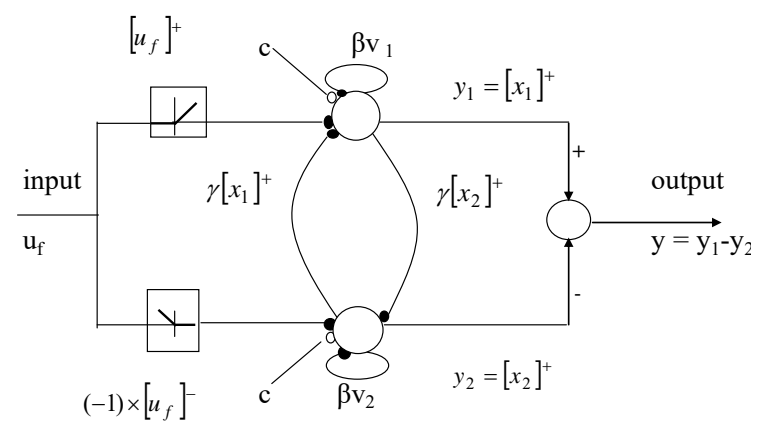

Fig. 1 Oscillator model

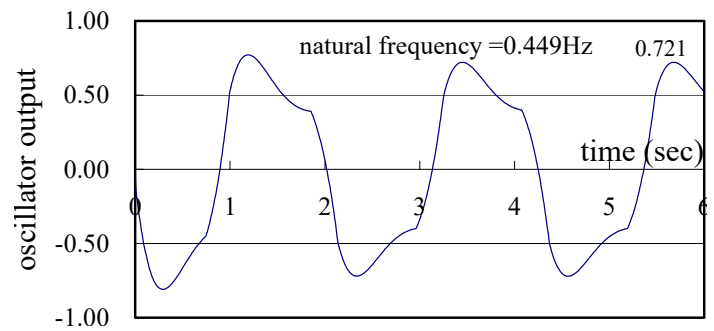

Fig. 2 A sample output from the oscillator $\left(\tau_{1}=0.25, \tau_{2}=0.50, \beta=2.5\right.$, $\gamma=2.5, c=1.5)$

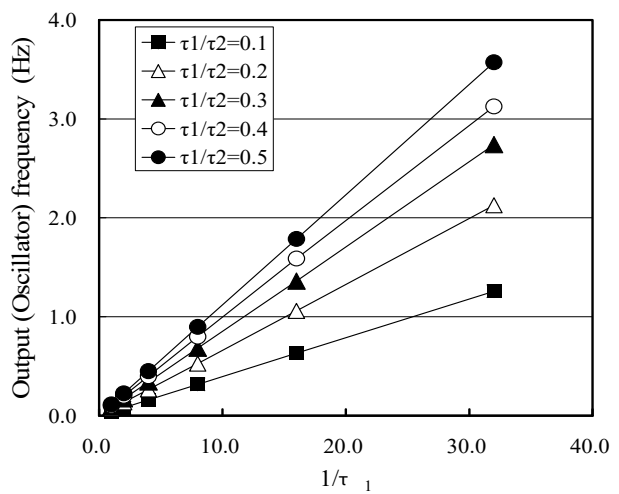

Fig. 3 Output frequency of the oscillator plotted against $1 / \tau_{1}(\beta=2.5$, $\gamma=2.5, c=1.5$ )

When an oscillatory input is applied to the neural-oscillator, the oscillator can entrain the input, locking in the input frequency. This is illustrated in Fig. 4, which shows the output of the oscillator as the size of the input signal is increased. Based on this figure, it can be confirmed that the oscillator is not entrained for small input (top graph), and that the oscillator is almost entrained when the input is larger in the middle graph. Moreover, it can be seen from the lower graph that the oscillator locked in the input frequency.

TABLE I

EVALUATION EQUATION FOR NATURAL FREQUENCY $(\beta=\gamma=2.5, \mathrm{c}=1.5)$

\begin{tabular}{cc}
\hline \hline$\tau_{1} / \tau_{2}$ & Natural frequency $(\mathrm{Hz})$ \\
\hline 0.1 & $f=0.0395 \times 1 / \tau_{1}$ \\
0.2 & $f=0.0665 \times 1 / \tau_{1}$ \\
0.3 & $f=0.0850 \times 1 / \tau_{1}$ \\
0.4 & $f=0.0991 \times 1 / \tau_{1}$ \\
0.5 & $f=0.1121 \times 1 / \tau_{1}$ \\
\hline
\end{tabular}

TABLE II

EVALUATION EQUATION FOR NATURAL FREQUENCY $(\beta=\gamma=1.5, \mathrm{c}=1.5)$

\begin{tabular}{cc}
\hline \hline$\tau_{1} / \tau_{2}$ & Natural frequency $(\mathrm{Hz})$ \\
\hline 0.1 & $f=0.0454 \times 1 / \tau_{1}$ \\
0.2 & $f=0.0689 \times 1 / \tau_{1}$ \\
0.3 & $f=0.0861 \times 1 / \tau_{1}$ \\
0.4 & $f=0.1012 \times 1 / \tau_{1}$ \\
0.5 & $f=0.1125 \times 1 / \tau_{1}$ \\
\hline \hline
\end{tabular}

Numerical simulations were carried out taking into consideration various combinations of the parameters of the neural-oscillator model, with the particular focus on the lock-in phenomenon. Fig. 5 shows the relationship between input and output (oscillator) frequencies. Based on this figure, it can be understood that there is the positive slope in which output oscillator frequency is nearly equal to input frequency, though oscillator frequency is just set up to be $1.000 \mathrm{~Hz}$. These analytical results clearly demonstrate that the neural-oscillator might be one of the useful models to explain the synchronization (the lock-in phenomenon) of a fairly high part of all pedestrians being on the bridge.

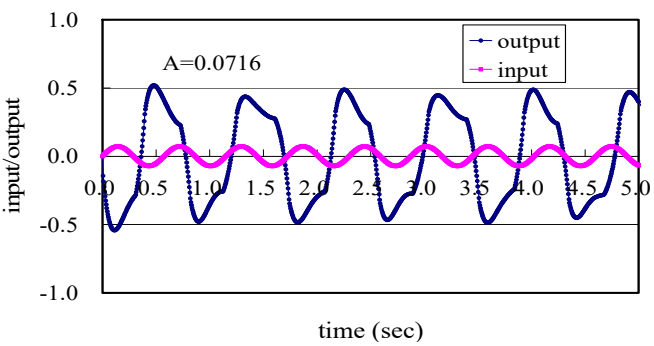

(a) Input amplitude $=0.0716$

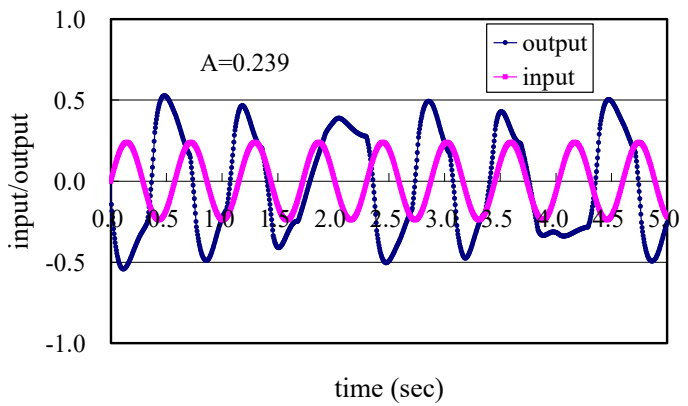

(b) Input amplitude $=0.239$ 


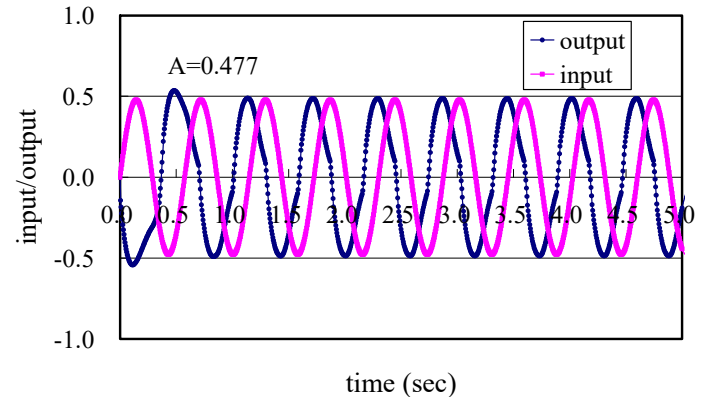

(c) Input amplitude $=0.477$

Fig. 4 Effect of increasing input signal on oscillator output $\left(\tau_{1}=0.1\right.$, $\tau_{2}=0.2, \beta=\gamma=2.5, c=1.0$, natural frequency of the oscillator $=1.122 \mathrm{~Hz}$, output frequency $=1.738 \mathrm{~Hz}$ )

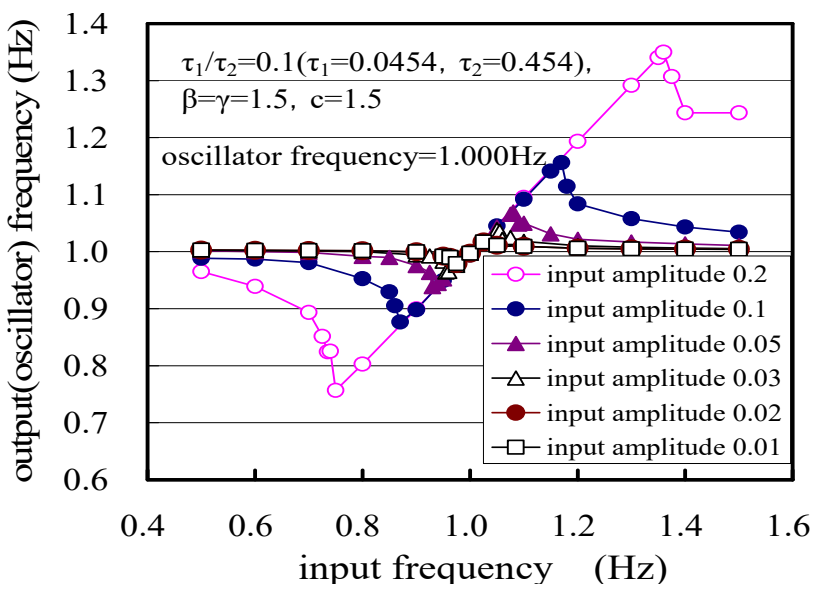

Fig. 5 Relationship between input and oscillator frequencies $\left(\tau_{1} / \tau_{2}=0.1\right)$

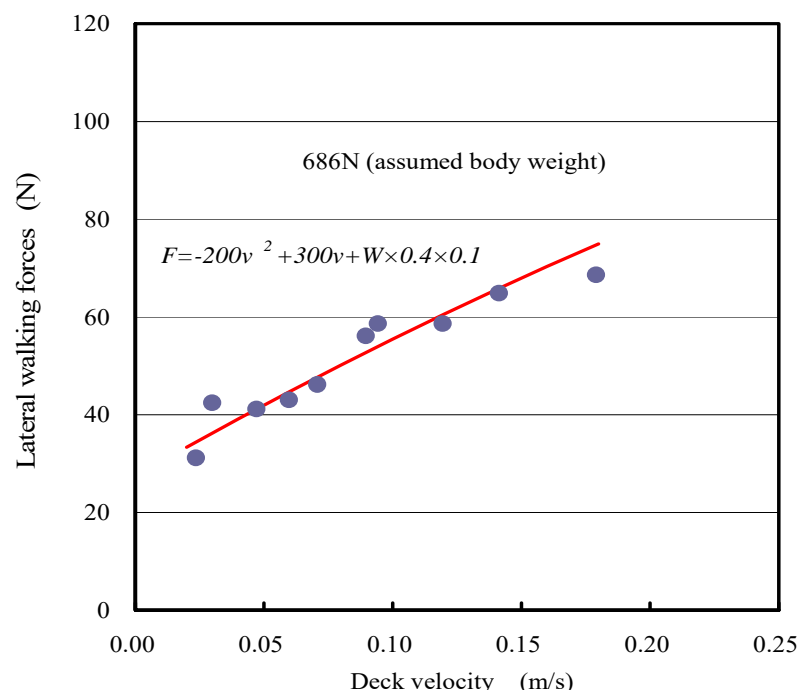

Fig. 6 Relationship between newly proposed lateral walking force and deck velocity applied for the Imperial College test results

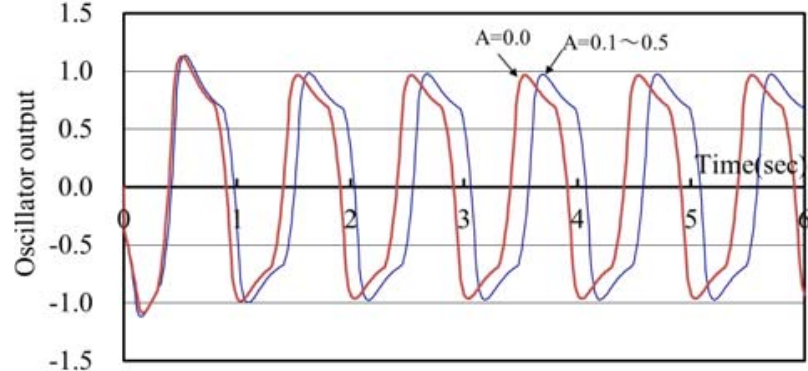

Fig. 7 Oscillator output $\left(\tau_{1} / \tau_{2}=0.1, \beta=\gamma=1.5, c=1.5\right.$, natural frequency of the oscillator $=1.00 \mathrm{~Hz}$

\section{A Newly Proposed Lateral WaLking ForCe}

A design formula of

$$
F=300 v
$$

has been proposed [1], based on the measurements on the Millennium Bridge, where $v$ is the local bridge lateral velocity amplitude $(\mathrm{m} / \mathrm{s})$ and $F$ is the effective force per person $(\mathrm{N})$, occurring at the natural frequency of the bridge. But, it must be noted that the lateral walking force due to this formula does not be generated if the initial deck velocity is not given and increases infinitely according to the increase of velocity amplitude. By the way, the vertical component of the dynamic walking forces is applied at the footfall frequency (typically around $2 \mathrm{~Hz}$ ) and is about $40 \%$ of their body weight. The lateral component is applied at half the footfall frequency and on a stationary surface is about 10 times smaller than the vertical component. Therefore, in this paper, the following equation of

$$
F=-200 v^{2}+300 v+W \times 0.4 \times 0.1
$$

is proposed in order to estimate the lateral walking force per person $(\mathrm{N})$, where $W$ is body weight.

The lateral walking force using (8) is compared with the Imperial College test results as shown in Fig. 6, in which the body weight of person is assumed to be $686 \mathrm{~N}$. It can be seen from this figure that the lateral walking force generated by pedestrians is the fairly good agreement in comparison with the results measured at the Imperial College.

\section{DyNAMIC RESPONSE ANALYSES FOR THE BRIDGE MODEL}

\section{A. Bridge Model}

A pedestrian bridge with the span of $50 \mathrm{~m}$ was selected as an analytical model. The $1^{\text {st }}$ symmetric lateral natural frequency of this bridge model, which is assumed to be simply supported in lateral direction, is just to be $1.000 \mathrm{~Hz}$. The weight per unit length is $14.7 \mathrm{kN} / \mathrm{m} / \mathrm{Br}$. and the logarithmic decrement is assumed to be 0.02 .

\section{B. Dynamic Response Analyses for the Bridge Model Caused by the Crowd}

The maximum output of CASE-151515 is almost 1.0 even if an external oscillation with any amplitude is inputted to the oscillator as shown in Fig. 7. Accordingly, it is very easy to 
convert the neural-oscillator output to the pedestrian lateral force by using (8). For this reason, CASE-151515 is considered in this research.

The movement at the Millennium Bridge was clearly caused due to the synchronization of lateral footfall forces within a large crowd of pedestrians on the bridge. Therefore, in this section, the dynamic response analysis was carried out taking into account the neural-oscillator model (CASE-151515) with the natural frequency of $0.950 \sim 1.050 \mathrm{~Hz}$ in good order. Assuming the pedestrians (231 person's row) are uniformly distributed over the whole span as shown in Fig. 8, the longitudinal distances between the pedestrians are assumed to be $1.0 \mathrm{~m}$, respectively.

Fig. 9 shows the time history displacement at the center of the span obtained by the dynamic response analysis. It can be seen from this figure that the catastrophic vibration start rapidly

231 persons (longitudinal distances: $1 \mathrm{~m}$ )

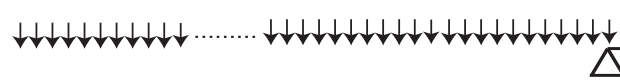

$\ldots 0.960 \mathrm{~Hz}, 0.950 \mathrm{~Hz}, 1.050 \mathrm{~Hz} \ldots 1.000 \mathrm{~Hz} \ldots 0.950 \mathrm{~Hz}$

\section{$\triangle$}

starting point

response is not almost observed up to 95 seconds.

Fig. 10 shows the frequency which is obtained by zero-crossing method for the time history generalized walking lateral force. Based on this figure, it can be seen that the dominant frequency which is scattered widely until 95 seconds is beginning to converge slowly and has reached rapidly to around $1.010 \mathrm{~Hz}$ at the time of 115 seconds.

For reference, the change of oscillator frequency is figured out for the oscillator model with the original frequency of 0.950 $\mathrm{Hz}$, by means of zero-crossing method for the time history oscillator output. This result is shown in Fig. 11. It can be seen from this figure that the each oscillator frequency is almost entrained to be equal to around $1.000 \mathrm{~Hz}$ which is the lateral natural frequency of the bridge according to the increase of the displacement although the oscillator shows complicated behavior at the beginning of the vibration.

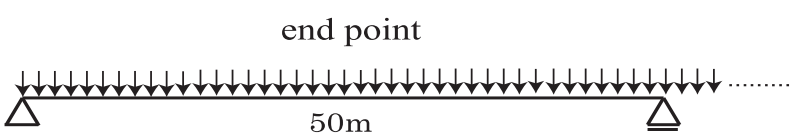

Fig. 8 Assumed pedestrians uniformly distributed over the whole span

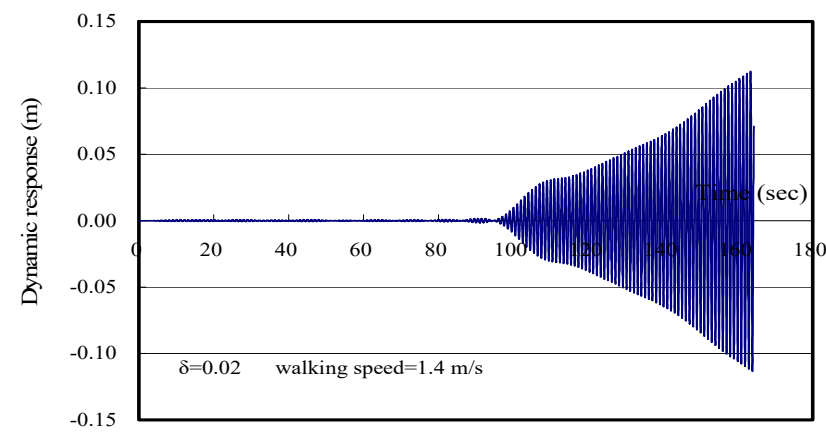

Fig. 9 Time history displacement at the center of the span obtained by the dynamic response analysis

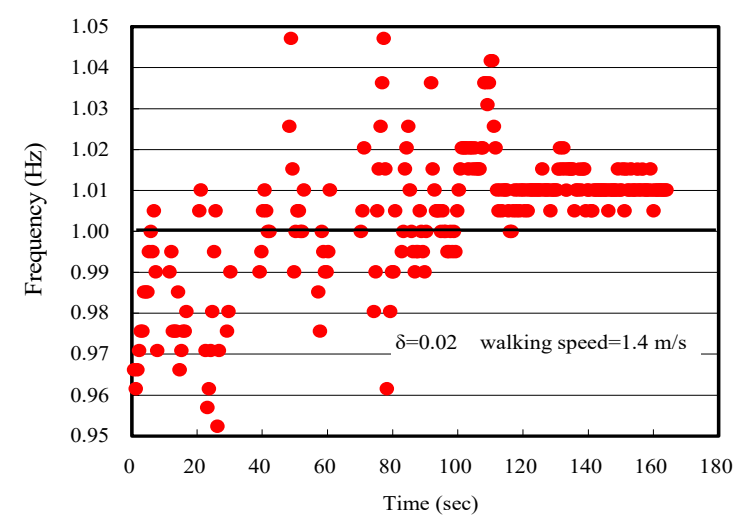

Fig. 10 Frequency of generalized walking lateral force

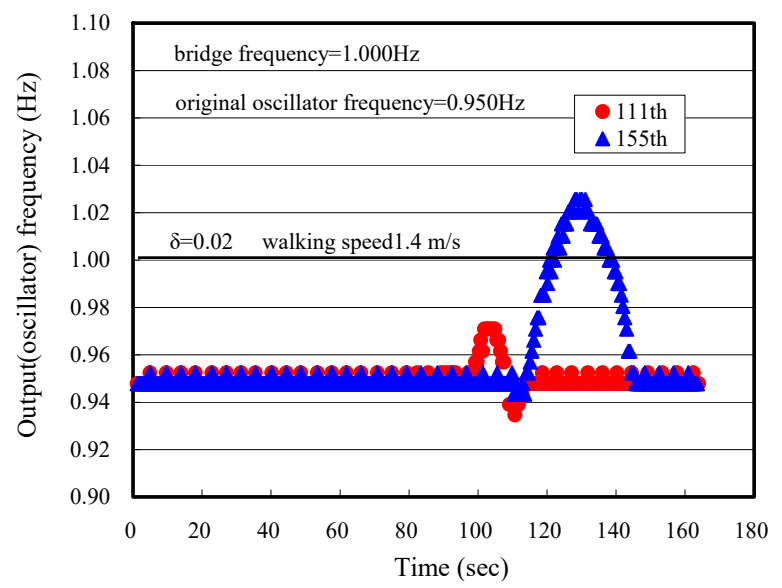

Fig. 11 Output (oscillator) frequency (original frequency $=0.950 \mathrm{~Hz}$ )

\section{V.FuLl SCALE MEASUREMENTS AND COMPARISON WITH DYNAMIC RESPONSE ANALYSES}

\section{A. Existing Pedestrian Suspension Bridge}

An existing suspension pedestrian bridge shown in Fig.12 which is constructed in an amusement park located in Osaka prefecture is selected for full scale measurements and dynamic response analyses.

Damped free vibration test induced by five people is carried out in state of windless time to decrease the aerodynamic damping effect. It is confirmed that the fundamental natural 
lateral frequency (symmetric 1st mode frequency) is $0.8545 \mathrm{~Hz}$ and the logarithmic decrement corresponding to this mode is 0.1242 based on the time history acceleration and the result of FFT analysis. It is appended that the amplitude dependency of the structural damping is negligible.

\section{B. Full Scale Measurements}

The test is conducted under the walking load of five people. The weight of five people is $637 \mathrm{~N}$ (male), $598 \mathrm{~N}$ (male), $470 \mathrm{~N}$ (female), $745 \mathrm{~N}$ (male) and $568 \mathrm{~N}$ (male), respectively. Five pedestrians were making a group with $1 \mathrm{~m}$ distance walking from end to end through the center span length with $62.4 \mathrm{~m}$, tried to synchronize their steps in response to the sound of an electronic metronome with frequency of $1.65 \mathrm{~Hz}$, which is $3.5 \%$ smaller compared with the doubled frequency of 0.8545 $\mathrm{Hz}(1.70 \mathrm{~Hz})$. This experiment case is described such as CASE-W165P5. The acceleration at the middle point of the girder and that of the right lumber of head pedestrian is measured. These obtained data are filtered with the frequency range of between $0.5-1.2 \mathrm{~Hz}$.

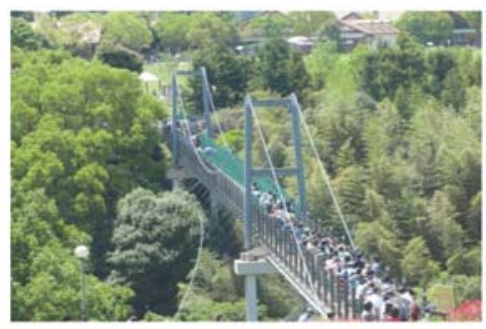

Fig. 12 Existing suspension pedestrian bridge

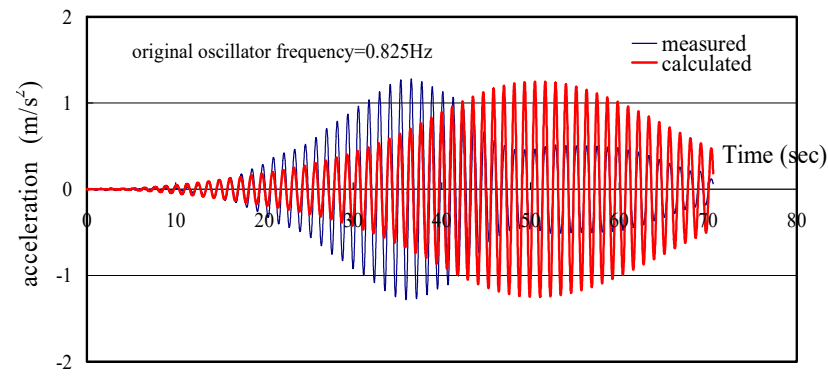

Fig. 13 Relationship between measured and calculated accelerations (CASE-W165P5)

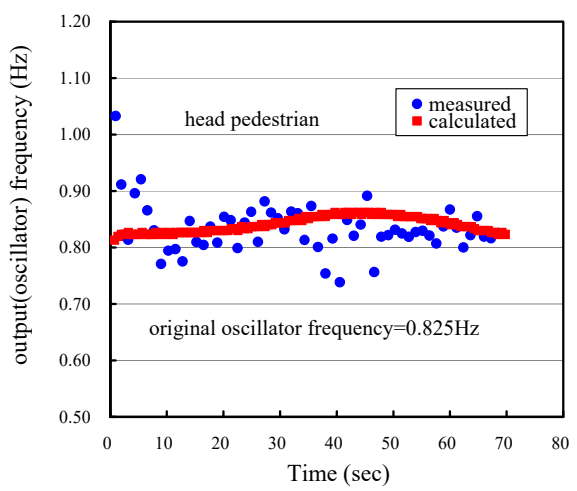

Fig. 14 Relationship between measured and calculated oscillator frequencies (CASE-W165P5)

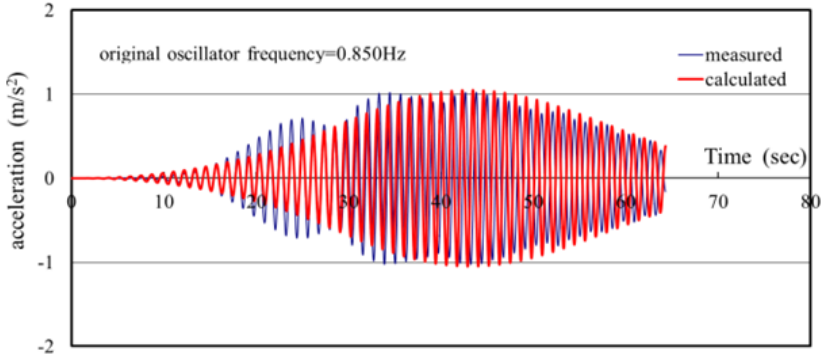

Fig. 15 Relationship between measured and calculated accelerations (CASE-W170P5)

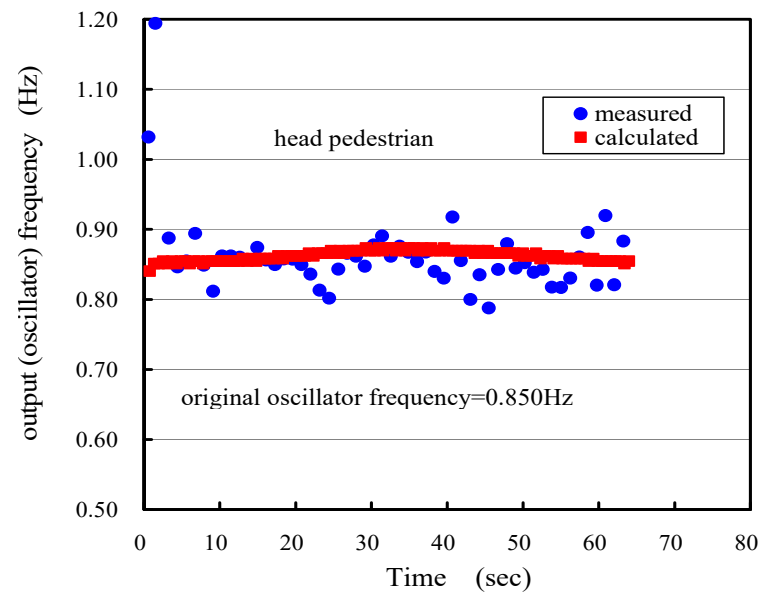

Fig. 16 Relationship between measured and calculated oscillator frequencies (CASE-W170P5)

\section{Comparison with Analytical and Experimental Results}

The dynamic response analysis was carried out taking into account the neural-oscillator model (CASE-151515) under the condition that the ratio of $\tau_{1} / \tau_{2}$ is 0.1 for the case of CASE-W165P5. The lateral walking force per person $(\mathrm{N})$ as shown in (8) is considered. The logarithmic decrement of 0.1242 is selected for the fundamental $1^{\text {st }}$ symmetric mode based on full scale measurements. Fig. 13 shows the acceleration response at the middle point of the suspension bridge obtained from the analysis and experiment in case of CASE-W165P5. Based on this figure, it is found that the acceleration response from analysis is nearly similar to the experiment result with the maximum acceleration of $1.2 \mathrm{~m} / \mathrm{s}^{2}$ (converted displacement is about $4.2 \mathrm{~cm}$ ); however, the analysis result is growing a bit slowly and the experiment result at the time of 40 seconds is drastically dropping to a low value because pedestrians off-balanced their walking steps due to the rapid increase of the girder amplitude. This situation can be understood from Fig. 14 which shows the lateral frequencies measured at the right lumber of the head pedestrian compared with the calculated oscillator frequency which is obtained by zero-crossing method for the time history oscillator output.

The dynamic response analysis was also conducted for the case of CASE-W170P5 which was tried to synchronize five people's steps in response to sound of electronic metronome with frequency of $1.70 \mathrm{~Hz}$. Fig. 15 shows the acceleration response at the middle point of the suspension bridge obtained 
from the analysis and experiment in case of CASE-W170P5. Based on this figure, it is found that the acceleration response from analysis is nearly equal to the experiment result with the maximum acceleration of $1.0 \mathrm{~m} / \mathrm{s}^{2}$. Fig. 16 shows the lateral frequencies measured at the right lumber of the head pedestrian compared with the calculated oscillator frequency which is obtained by zero-crossing method for the time history oscillator output. Based on this figure, it might be said that the measured lateral frequency varies between $0.78 \mathrm{~Hz}$ and 0.90 $\mathrm{Hz}$ as beat wave, although the calculated oscillator frequency shows gradual slight change between $0.82 \mathrm{~Hz}$ and $0.86 \mathrm{~Hz}$.

\section{CONCLUDING REMARKS}

This paper deals with dynamic response analyses for human-induced lateral vibration on congested pedestrian bridge taking into account the neural-oscillator model. The results of this paper are summarized below:

1) The new evaluation formula which is proportional to square value of the deck velocity is proposed in order to convert the oscillator output to the pedestrian lateral force.

2) The catastrophic vibration due to the crowd is reappeared analytically assuming the pedestrians (231 person's row) are uniformly distributed over the whole span of bridge model with the length of $50 \mathrm{~m}$.

3) As compared with full scale measurements for the existing pedestrian suspension bridge, it is confirmed that the acceleration response from analysis is nearly similar to the experiment result with the maximum acceleration of 1.2 $\mathrm{m} / \mathrm{s}^{2}$ (converted displacement is about $4.2 \mathrm{~cm}$ ).

\section{REFERENCES}

[1] Dallard, P., Fitzpatrick, A. J., Flint, A., Le Bourva, S., Low, A., Ridsdill Smith, R. M. and Willford, M. ,"The London Millennium Footbridge", The Structural Engineer, vol.79, no.22, 2001, pp.17-33.

[2] Fujino, Y., Pacheco, M. B., Nakamura, S. and Pennung, W., "Synchronization of Human Walking Observed during Lateral Vibration of a Congested Pedestrian Bridge", Earthquake Engineering and Structural Dynamics, vol.22, 1993, pp.741-758.

[3] Matsuoka, K., "Sustained Oscillations Generated by Mutually Inhibiting Neurons with Adaptations", Biological Cybernetics, 52, 1985, pp.367-376.

[4] Williamson, M., "Neural Control of Rhythmic Arm Movements", Neural Networks, 11, 1998, pp.1379-1394.

[5] Yoneda, M. and Fukae, M., "A Fundamental Study on Neural-oscillator-based Algorithm for Human-induced Lateral Vibration on Congested Pedestrian Bridges", Journal of Structural Engineering, 54A, 2008, pp.218-227. (in Japanese). 